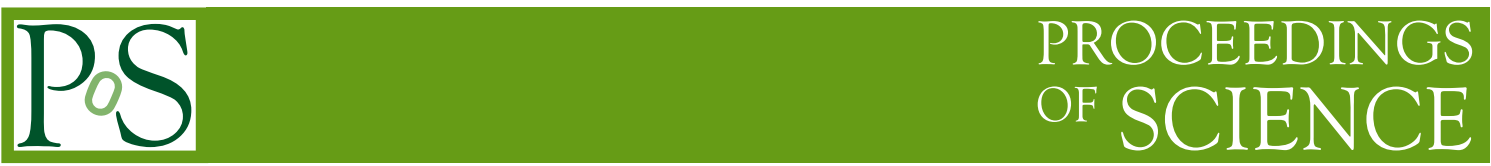

\title{
The $D_{s_{1}}(2460)$ as a molecular state in a constituent quark model
}

\author{
D.R. Entem* \\ Grupo de Física Nuclear and IUFFyM, Universidad de Salamanca \\ E-mail: entemeusal.es
}

\section{P.G. Ortega}

Grupo de Física Nuclear and IUFFyM, Universidad de Salamanca e-mail: pgortega@usal.es

\section{F. Fernández}

Grupo de Física Nuclear and IUFFyM, Universidad de Salamanca

E-mail: fdz@usal.es

The $D_{s} P$-wave mesons have been revealed as an excellent system to test low momentum QCD. In particular the low masses of the $D_{s_{0}}^{*}(2317)$ and $D_{s_{1}}(2460)$ states represents a challenge for phenomenological models. In the present work we use the chiral constituent quark model to couple the $c \bar{s} 1^{+}$components with $D^{*} K, D_{s}^{*} \eta, D_{s} \omega$ and $D K^{*}$ molecular components microscopically with the same ${ }^{3} P_{0}$ model used to study strong decays. We obtain a state below the $D^{*} K$ threshold explaining the low mass of the $D_{s_{1}}(2460)$. A state above threshold is also found which could be identified with the $D_{s_{1}}(2536)$. In the $0^{+}$sector we perform a similar calculation coupling $c \bar{s}$ components with $D K$ molecular components. In this case no molecular bound state is found.

Sixth International Conference on Quarks and Nuclear Physics,

April 16-20, 2012

Ecole Polytechnique, Palaiseau, Paris

\footnotetext{
*Speaker.
} 


\section{Introduction}

Experiments in the last decade has challenge our understanding of meson spectroscopy. One of the examples has been the discovery of the $D_{s} P$-wave mesons. In 2003 the BABAR Collaboration observed the $D_{s_{0}}^{*}$ (2317) state [1], soon confirmed by the CLEO Collaboration [2] which also reported a new state called $D_{S_{1}}(2460)$. Later on, the Belle Collaboration measured both states $[3,4]$ and found results consistent with the spin-parity assignments $J^{P}=0^{+}$for the $D_{s_{0}}(2317)$ and $J^{P}=1^{+}$for the $D_{S_{1}}(2460)$. These data differs strongly from the expectation of Heavy Quark Symmetry (HQS) and the previously measured masses of the $D_{s_{1}}(2536)$ and the $D_{s_{2}}^{*}(2573)$. Assuming HQS one expects the $c$ quark as a frozen degree of freedom, so the relevant quantum numbers would be the total angular momentum of the $\bar{s}$ antiquark $j_{q}=L+s_{q}$. As the angular momentum of the light antiquark is 1 this gives two possible states $j_{q}=1 / 2$ and $j_{q}=3 / 2$. Together with the spin of the $c$ quark would give two possible multiplets, namely a $0^{+}, 1^{+}$and a $1^{+}, 2^{+}$. So one would expect the two sates measured in 2003 almost degenerate and close to the previously measured states.

\section{The constituent quark model}

The chiral constituent quark model used in the present work has been describe in Ref. [5], so we will only present its most important features.

The model is based on the symmetries of QCD and in particular in its spontaneously broken chiral symmetry. This effect makes the light quarks to acquire a constituent quark mass and interact through pseudo-Goldstone bosons, given by the pseudo-scalar meson octet. Besides this, QCD non-perturbative effects are included through a color confinement interaction which we take as a linear screened potential. QCD perturbative effects are included with gluon interactions. In the $q \bar{q}$ case besides boson exchange interaction also annihilation diagrams are included. All the model parameters used in this work can be found in Ref. [6].

With the same interquark interaction we use the Resonating Group Method to build the interaction between mesons computing the diagrams of the first row of Fig. 1. For the interacting mesons we use the wave functions solutions of the $q \bar{q}$ Hamiltonian. In the present work there is no antisymmetry between quarks of different mesons and so only direct diagrams are included. However we have to take into account rearrangement processes and so the inclusion of the $D^{*} K$ $\left(D K^{*}\right)$ implies the inclusion of the $D_{s}^{*} \eta\left(D_{s} \omega\right)$ channels. Color interactions doesn't apply between colorless object but they contribute to rearrangement diagrams which are shown in the second row of Fig 1. In Table 1 we give a summary of the different terms that contributes to the interaction and mixing between the two meson states relevant in the $1^{+}$sector.

\section{Coupling $q \bar{q}$ and $q \bar{q} q \bar{q}$ components}

We assumed the hadronic state to be a combination of one and two-meson states in the form

$$
|\Psi\rangle=\sum_{\alpha} c_{\alpha}\left|\psi_{\alpha}\right\rangle+\sum_{\beta} \chi_{\beta}(P)\left|\phi_{A} \phi_{B} \beta\right\rangle
$$



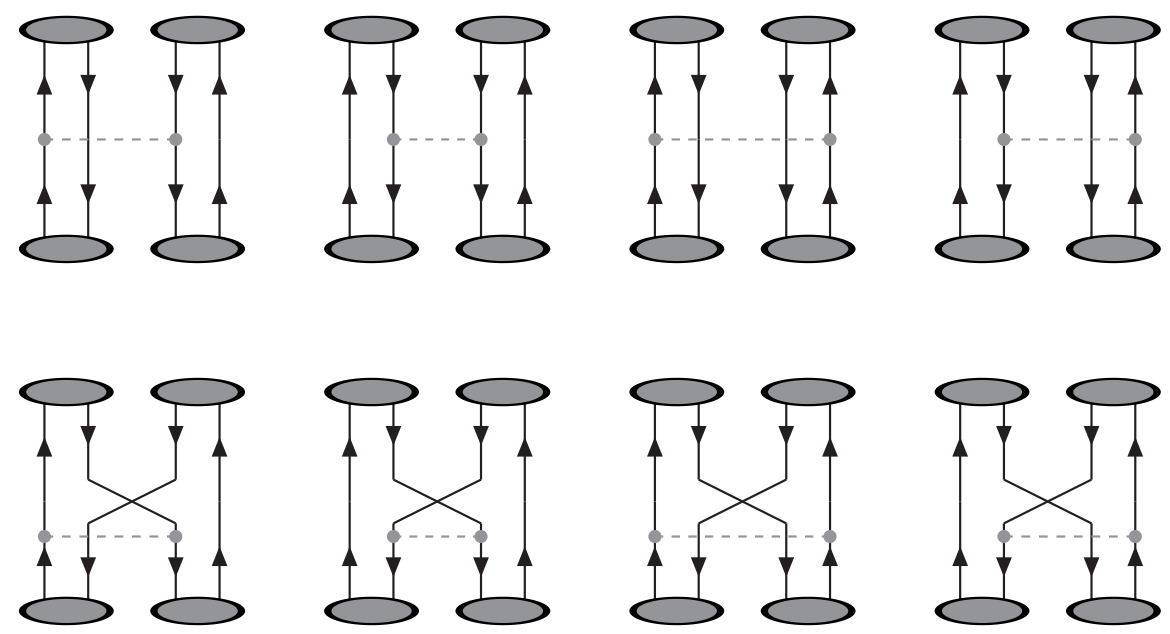

Figure 1: Diagrams that contributes to the interaction and mixing between two meson states. The first row shows the interaction terms and the second row the rearrangement processes.

\begin{tabular}{c|cccc}
\hline \hline & $D^{*} K$ & $D_{s}^{*} \eta$ & $D_{s} \omega$ & $D K^{*}$ \\
\hline$D^{*} K$ & $\sigma+a n h$ & $\kappa+r e a+a n h$ & $K+$ rea & $\pi+a n h$ \\
$D_{s}^{*} \eta$ & $\kappa+r e a+a n h$ & $\sigma+a n h$ & - & $K+$ rea + anh \\
$D_{s} \omega$ & $K+$ rea & - & $\sigma$ & $\kappa+$ rea \\
$D K^{*}$ & $\pi+a n h$ & $K+$ rea + anh & $\kappa+$ rea & $\sigma+a n h$ \\
\hline \hline
\end{tabular}

Table 1: Different terms that contributes to the interaction and mixing between different two meson states. Here anh means annihilation diagrams and rea rearrangement diagrams.

where $\left|\psi_{\alpha}\right\rangle$ are $q \bar{q}$ eigenstates of the two body Hamiltonian, $\phi_{M}$ are $q \bar{q}$ eigenstates describing the $A$ and $B$ mesons, $\left|\phi_{A} \phi_{B} \beta\right\rangle$ is a two meson state with $\beta$ quantum numbers and $\chi_{\beta}(P)$ is the relative wave function between the two mesons.

The coupling between $q \bar{q}$ and $q \bar{q} q \bar{q}$ configurations which describes meson strong decays is a non-perturbative process that have not been explained from first principles. Usually phenomenological models are used and one of the most popular is the ${ }^{3} P_{0}$ model [7]. This is the one we use in the present work and is describe from the Hamiltonian

$$
\mathscr{H}=g \int d^{3} x \bar{\psi}(x) \psi(x)
$$

which defines the transition potential $h_{\beta \alpha}(P)$

$$
\left\langle\phi_{M_{1}} \phi_{M_{2}} \beta|\mathscr{H}| \psi_{\alpha}\right\rangle=P h_{\beta \alpha}(P) \delta^{(3)}\left(\vec{P}_{\mathrm{cm}}\right)
$$


The ${ }^{3} P_{0}$ model introduces only one parameter usually given by the dimensionless constant $\gamma=\frac{g}{2 m}$ and we fit its value to the $\psi(3770) \rightarrow D \bar{D}$ decay [6].

The system is govern by the coupled-channel equations

$$
\begin{aligned}
c_{\alpha} M_{\alpha}+\sum_{\beta} \int h_{\alpha \beta}(P) \chi_{\beta}(P) P^{2} d P & =E c_{\alpha} \\
\sum_{\beta} \int H_{\beta^{\prime} \beta}\left(P^{\prime}, P\right) \chi_{\beta}(P) P^{2} d P+\sum_{\alpha} h_{\beta^{\prime} \alpha}\left(P^{\prime}\right) c_{\alpha} & =E \chi_{\beta^{\prime}}\left(P^{\prime}\right)
\end{aligned}
$$

where $M_{\alpha}$ are the masses of the bare $\alpha$ mesons and $H_{\beta^{\prime} \beta}$ is the RGM Hamiltonian for the two meson states obtained from the $q \bar{q}$ interaction. Following Ref. [8] the solution is given by the $T$-matrix

$$
T^{\beta^{\prime} \beta}\left(P^{\prime}, P ; E\right)=T_{V}^{\beta^{\prime} \beta}\left(P^{\prime}, P ; E\right)+\sum_{\alpha, \alpha^{\prime}} \phi^{\beta^{\prime} \alpha^{\prime}}\left(P^{\prime} ; E\right) \Delta_{\alpha^{\prime} \alpha}^{-1}(E) \phi^{\alpha \beta}(P ; E)
$$

with $T_{V}^{\beta^{\prime} \beta}\left(P^{\prime}, P ; E\right)$ the $T$ matrix of the RGM potential excluding the coupling to the $q \bar{q}$ pairs,

$$
\Delta_{\alpha^{\prime} \alpha}^{-1}(E)=\left(\left(E-M_{\alpha}\right) \delta^{\alpha^{\prime} \alpha}+\mathscr{G}^{\alpha^{\prime} \alpha}(E)\right)^{-1}
$$

is the propagator of the mixed state,

$$
\phi^{\beta \alpha}(P ; E)=h_{\beta \alpha}(P)-\sum_{\beta^{\prime}} \int \frac{T_{V}^{\beta \beta^{\prime}}(P, q ; E) h_{\beta^{\prime} \alpha}(q)}{q^{2} / 2 \mu-E} q^{2} d q
$$

is the vertex function dressed by the two-meson interaction and

$$
G^{\alpha^{\prime} \alpha}(E)=\sum_{\beta} \int \frac{\phi_{\alpha^{\prime} \beta}(q, E) h_{\beta \alpha}(q)}{q^{2} / 2 \mu-E} q^{2} d q
$$

is the mass shift induced by meson loops.

The physical states are given by poles of the $T$-matrix in the second Riemann sheet given by the solutions of the equation

$$
\left|\Delta_{\alpha^{\prime} \alpha}(\bar{E})\right|=\left|\left(\bar{E}-M_{\alpha}\right) \delta^{\alpha^{\prime} \alpha}+\mathscr{G}^{\alpha^{\prime} \alpha}(\bar{E})\right|=0
$$

with $\bar{E}$ the pole position.

\section{Results}

\subsection{The $1^{+}$sector}

In the $1^{+}$sector the naive quark model predicts two states with masses $2571 \mathrm{MeV}$ in the ${ }^{3} P_{1}$ partial wave and $2576 \mathrm{MeV}$ in the ${ }^{1} P_{1}$. The closest two meson thresholds in this sector are the $D^{*} K$ at $2504 \mathrm{MeV}$, the $D_{s}^{*} \eta$ at $2660 \mathrm{MeV}$, the $D_{s} \omega$ at $2751 \mathrm{MeV}$ and the $D K^{*}$ at $2764 \mathrm{MeV}$. We perform a couple channel calculation in the framework previously described and find two resonances, one below and one above the $D^{*} K$ threshold. Their masses and channel probabilities are given in Table 2. 


\begin{tabular}{cccc}
\hline \hline Mass $(\mathrm{MeV})$ & $c \bar{s}\left({ }^{1} P_{1}\right)$ & $c \bar{s}\left({ }^{3} P_{1}\right)$ & molecule \\
\hline \hline 2481.1 & 39.5 & 24.6 & 35.9 \\
$2509.9-\mathrm{i} 0.1$ & 14.9 & 30.9 & 54.2 \\
\hline \hline
\end{tabular}

Table 2: Mass and channel probabilities (in \%) of the two resonances found in the $1^{+}$sector.

We find a state below threshold that can be assigned to the $D_{s_{1}}(2460)$ although we get a mass which is $20 \mathrm{MeV}$ above the physical mass. In this case

$$
\left(\frac{\operatorname{Prob}\left({ }^{1} P_{1}\right)}{\operatorname{Prob}\left({ }^{3} P_{1}\right)}\right)_{D_{s}(2460)}=0.62
$$

which is close to the 0.5 value for a $j_{q}=1 / 2$ state.

The state above threshold has an opposite sign between the ${ }^{1} P_{1}$ and ${ }^{3} P_{1}$ amplitudes and

$$
\left(\frac{\operatorname{Prob}\left({ }^{1} P_{1}\right)}{\operatorname{Prob}\left({ }^{3} P_{1}\right)}\right)_{D_{s}(2526)}=2.07
$$

which is close to the 2 value for a pure $j_{q}=3 / 2$ state. The state $D_{s_{1}}(2536)$ has many properties that are consistent with this admixture. This was analyzed in a previous work [9] where a phenomenological coupling with a tetraquark state was used and the properties of the state were well described.

If we allow the bare masses to vary we can fit the mass of the $D_{s_{1}}(2460)$ and the $D_{s_{1}}(2536)$ using a bare mass of $2533 \mathrm{MeV}$ for the ${ }^{3} P_{1}$ state and 2624 for the ${ }^{1} P_{1}$. The width of the $D_{s_{1}}(2536)$ is in this case $12 \mathrm{MeV}$ indicating that the mixture between $j_{q}=1 / 2$ and $j_{q}=3 / 2$ is too high.

\subsection{The $0^{+}$sector}

In the $0^{+}$sector we perform a similar calculation including the $D K$ threshold. The bare mass of the ${ }^{3} P_{0}$ state is $2510 \mathrm{MeV}$. We only find a resonance at $M=2504-i 83.6 \mathrm{MeV}$ so it makes evident that this mechanism can not explain the low mass of the $D_{s_{0}}(2317)$. Other possible mechanisms has been proposed by Lakhina [10] due to one loop contributions [11] that gives big mass shifts in this partial wave while the contribution in the $1^{+}$sector is not so important [12].

This work has been partially funded by Ministerio de Ciencia y Tecnología under Contract Nos. FPA2010-21750-C02-02, by the European Community-Research Infrastructure Integrating Activity 'Study of Strongly Interacting Matter' (HadronPhysics2 Grant No. 227431) and by the Spanish Ingenio-Consolider 2010 Program CPAN (CSD2007-00042).

\section{References}

[1] B. Aubert et al. (BABAR Collaboration), Phys. Rev. Lett. 90, 242001 (2003).

[2] D. Besson et al. (CLEO Collaboration), Phys. Rev. D 68, 032002 (2003).

[3] P. Krokovny et al. (Belle Collaboration), Phys. Rev. Lett. 91, 262002 (2003).

[4] Y. Mikami et al. (Belle Collaboration), Phys. Rev. Lett. 92, 012002 (2004). 
[5] J. Vijande, F. Fernández, and A. Valcarce, J. Phys. G 31, 481 (2005).

[6] J. Segovia, A.M. Yasser, D.R. Entem, and F. Fernández, Phys. Rev. D 78, 114033 (2008).

[7] L. Micu, Nucl. Phys. B 10, 521 (1969).

[8] V. Baru et al., Eur. Phys. J. A 44, 93 (2010).

[9] J. Segovia, A.M. Yasser, D.R. Entem, and F. Fernández, Phys. Rev. D 80, 054017 (2009).

[10] O. Lakhina, and E.S. Swanson, Phys. Lett. B 650, 159 (2007).

[11] S.N. Gupta, and S.F. Radford, Phys. Rev. D 24, 2309 (1981).

[12] J. Segovia, C. Albertus, E. Hernandez, F. Fernandez, and D.R. Entem, arXiv:hep-ph/1203.4362 (2012). 